\title{
GLASSHOUSE - Smart Glasses zur Unterstützung von Logistikdienstleistungen
}

\author{
Oliver Thomas, Ingmar Ickerott, Lisa Berkemeier, Sebastian Werning, \\ Benedikt Zobel, Jannis Vogel, Christian Kaiser, Tobias Mollen-Ungru \\ und Thomas Neumann
}

GLASSHOUSE verfolgt die Unterstützung von Logistikdienstleistungen in der gesamten Wertschöpfungskette der Intralogistik mit Smart Glasses. Eine zentrale Annahme des Projekts ist, dass eine bedarfsgerechte Informationseinblendung in das Sichtfeld der Mitarbeiterinnen und Mitarbeiter bei der Erbringung der Dienstleistung unterstützt. Aktuellen Herausforderungen in der Logistik wird dadurch effektiv begegnet. Zu diesen zählt u.a. eine saisonbedingte hohe Mitarbeiterfluktuation, die oftmals mit kurzen Einlernphasen einhergeht. Das Besondere am Projekt GLASSHOUSE ist die facettenreiche Berücksichtigung und Erforschung notwendiger Faktoren wie Gebrauchstauglichkeit, Datenschutz, Ergonomie, Systemdesign und -aufbau für eine akzeptierte und nutzenstiftende digitale Lösung. Das Projektakronym lautet GLASSHOUSE, um durch die orthographische Nähe zum Lager (engl.: warehouse) die Verbindung mit Smart Glasses herzustellen.

\section{Die Logistikbranche im globalen, digitalen Aufbruch}

Die Logistik ist in Deutschland nach der Automobilwirtschaft und dem Handel der drittgrößte Wirtschaftszweig. Dabei verzeichnet die Logistikbranche seit 2015 einen stetig steigenden Umsatz: 279 Milliarden $€$ Umsatz mit ca. 3,2 Millionen Beschäftigten werden im Jahr 2019 erwartet (Bundesvereinigung Logistik e.V. 2018). Dabei zeichnet sich dieser bedeutende Dienstleistungszweig dadurch aus, dass komplexe und wissensintensive Prozesse ablaufen und eine Vielzahl an Mitarbeiterinnen und Mitarbeitern mit kurzen Einlernphasen eingesetzt wird. Dies stellt Unternehmen der Logistikbranche vor die Herausforderung, geeignete Fachkräfte zu finden. Vier von fünf Logistikunternehmen haben Schwierigkeiten, qualifiziertes Personal zu finden. Dabei betrifft dies alle Abteilungen und Funktionen, insb. herrscht ein Bedarf an Fachkräften für Umschlag und Lagerlogistik, der sich bedingt durch saisonale Schwankungen und eine hohe Mitarbeiterfluktuation verstärkt (Czernin und Schocke 2016). 
Automatisierungsbestrebungen und der Einsatz von Robotern und autonom fahrenden LKW können in Zukunft den Bedarf an Fachkräften reduzieren, nichtdestotrotz wird die Logistikbranche über die nächsten Jahreszehnte hinweg weiterhin personalintensive Prozesse enthalten. Dabei wird der Fachkräftebedarf auf Grund der Demografieentwicklung zunehmen. Technologische Innovationen können in der Logistik einen wertvollen Beitrag leisten, um ein geeignetes und substanziell besseres Arbeitsumfeld, vor allem auch für ältere Fachkräfte, zu schaffen (Schroven 2015). Die Digitalisierung ermöglicht weiterhin neuartige Services sowie Geschäftsmodelle. Diese können zu einer wahrgenommenen Differenzierung beim Kunden führen, sodass dem Kostendruck, bedingt durch die Globalisierung und eine gestiegene Transparenz im Markt durch digitale Angebote, aktiv begegnet wird. Zusätzliche Serviceleistungen können bspw. das Angebot ergänzen (Stölzle und Martin 2016).

In der Logistik sind Softwareanwendungen für logistische Prozesse wie der Lagerwirtschaft oder der Kommissionierung bereits Standard und die notwendige Informationsversorgung für die Mitarbeiter wird in entsprechenden Lagerhaltungssystemen vorgehalten (Helmke 2019). In der Aufbereitung dieser Daten und deren Nutzbarmachung sowie Visualisierung für eine effektive Mensch-Computer-Interaktion liegen ungenutzte Potenziale. Auf dem Weg zur „Logistik 4.0“ findet eine merkbar stärkere Vernetzung statt. Cyberphysische Systeme und autonome Roboterverbünde übernehmen selbstständig Aufgaben und daran angeknüpfte Softwareagenten priorisieren dabei Aufträge. In dieser richtungsweisenden Entwicklung benötigt der Mensch eine Schnittstelle, um als Akteur den Prozess prüfen und steuern zu können. Folgerichtig ist die Anknüpfung an mobile Assistenzsysteme eine logische Konsequenz (ten Hompel und Kerner 2015). Hier können Smart Glasses gegenüber anderen Geräteklassen wie Smartphones und Tablets einen Mehrwert leisten. Zum einen erhält der Nutzer Informationen direkt in das Sichtfeld eingeblendet, sodass er unmittelbar auf neue Informationen reagieren kann. Zum anderen hat der Anwender bzw. die Anwenderin beide Hände während des Arbeitsprozesses frei und kann bimanuelle Tätigkeiten mithilfe der Sprachein- und -ausgaben durchführen.

\section{Einsatzpotenziale von Smart Glasses in der Logistik}

Die Logistikbranche ist gekennzeichnet durch einen starken Preisdruck, hohe Qualitätsansprüche der Kunden, hohe Mitarbeiterfluktuation, einen durch den demografischen Wandel bedingten Fachkräftemangel, der u.a. durch ein anhaltendes Wachstum der Branche resultiert, sowie sich stetig verändernde Kundenwünsche und den Bedarf, flexibel auf diese zu reagieren (Kersten et al. 2017). Traditionelle Logistikprozesse kommen an ihre Grenzen und können die Informations- und Unterstützungsbedarfe der Logistikmitarbeiterinnen und -mitarbeitern nicht zufriedenstellend erfüllen. Ein enormes Potenzial zur Unterstützung der Logistikprozesse bieten Smart Glasses bzw. Datenbrillen. Diese blenden Information direkt in 
das Sichtfeld des Nutzers ein. Eine der bekanntesten Versionen ist die Google Glass, die im Jahr 2012 erschien und mit der sich industrietaugliche Anwendungsfälle realisieren lassen. Vorerst war die Datenbrille für den Konsumentenmarkt anvisiert. Hier zeigten sich jedoch wesentliche Akzeptanzprobleme, sodass Google das Produkt vom Markt nahm. Im Jahr 2017 machte Google schließlich das Nachfolgemodell der Google Glass ausschließlich für die Unternehmenslandschaft zugänglich. Die Smart-Glasses-Hersteller erkannten, dass ihre Technologien zwar nicht im Konsumentenmarkt akzeptiert, jedoch in der Industrie ihre Anwendung finden und dort bedingt durch den wahrgenommenen Nutzen stärker akzeptiert werden. Eine charakteristische Eigenschaft von Smart Glasses ist die Einblendung von Informationen direkt in das Sichtfeld. Dadurch erhält der Anwender stets einen direkten Informationszugriff und es sind keine störenden Aktivitäten notwendig, um Informationen abzurufen, die den Arbeitsprozess unterbrechen. Weiterhin hat der Anwender bei der Verwendung der Smart Glasses durch eine sprachbasierte Steuerung beide Hände frei.

In der Logistik ergeben sich eine Fülle verschiedenster Einsatzszenarien, um Arbeitsprozesse mit Smart Glasses zu unterstützen und zu digitalisieren. Während des Forschungsprojekts GLASSHOUSE wurden insg. 36 verschiedene Anwendungsfälle identifiziert, die in einer großen Bandbreite in Wertschöpfungsprozessen der Logistik angewendet werden können (Niemöller et al. 2017). Damit aus einem Anwendungsfall ein tatsächlicher Mehrwert resultiert, ist die Berücksichtigung von Einflussfaktoren auf die Akzeptanz und die Gebrauchstauglichkeit des Informationssystems entscheidend. Insg. können bis zu 40 Usability-Aspekte für Smart-Glasses-basierte Systeme herangezogen werden, die von Zobel et al. (2016) identifiziert wurden. Die Kenntnis von Usability-Eigenschaften ist insb. bei der Einführung von Smart-Glasses-basierten Systemen wichtig, um eine effektive Technologieauswahl, welche die Anforderungen an die betriebliche Situation berücksichtigt, treffen zu können.

Ein Einbezug relevanter Stakeholder erhöht die Akzeptanz der Nutzenden und verbessert die Technologieauswahl. Schließlich können diese bei Erprobungen erste Bewertungen abgeben und sind eine wichtige Informationsquelle zur Verbesserung von Smart-Glasses-basierten Systemen. Bei der Implementierung von entsprechenden Systemen ist eine intuitive und verständliche Gestaltung der grafischen Benutzeroberfläche (engl.: user interface, kurz: UI), Sprachsteuerung sowie weitere Interaktionsmöglichkeiten bspw. mittels Kontrollknöpfen unabdingbar, um eine Praxistauglichkeit und Akzeptanz der Anwender zu erzielen.

Aufgrund der Neuheit der Technologie existieren kaum Prinzipien bzw. Gestaltungsrichtlinien, die beim Design von Smart-Glasses-Applikationen heranzuziehen sind. Diese Tatsache verstärkt den integrativen Einbezug der Praxis in die Forschung und Gestaltung von entsprechenden Anwendungen. Eine gemeinschaftliche Zusammenarbeit zwischen Forschung und Praxis im Sinne der Konsortialforschung (Österle und Otto 2010), wie sie im GLASSHOUSE-Projekt verfolgt wurde, ermöglicht daher die Identifikation von Einsatzpotenzialen von Smart Glasses und deren Wirkung auf die logistischen Arbeitsprozesse. 


\section{GLASSHOUSE - Konzeption und Zielsetzung}

Das Erscheinen von neuen, performanteren Datenbrillen führte insb. in den Jahren 2015 und 2016 zu ersten Praxiserprobungen von Smart Glasses in der Logistikbranche und neuen kommerziellen Angeboten. Diese beschränkten sich jedoch ausschließlich auf den Prozess der Kommissionierung. Rückblickend ist dies begründet durch eine frühzeitige Identifikation von Verbesserungspotenzialen und die Notwendigkeit, die Kommissionierung zu optimieren. Die Logistikbranche erkannte die Vorteile eines Smart-Glasses-basierten Systems zur Unterstützung des Kommissionierungsprozesses gegenüber alternativen Methoden frühzeitig. Ungeachtet blieben jedoch weitere Funktionsbereiche wie die Einlagerung, Bereitstellung und der Umschlag, sodass eine ganzheitliche Betrachtung der gesamten Wertschöpfungsprozesse fehlte. Das Ziel vom dreijährigen Forschungsprojekt GLASSHOUSE ist es, diese Lücke in Wissenschaft und Praxis zu schließen, um Mitarbeiterinnen und Mitarbeiter in der Logistik in sämtlichen Prozessen ausnahmslos zu unterstützen und somit eine konsequent hohe Dienstleistungsproduktivität zu erzielen.

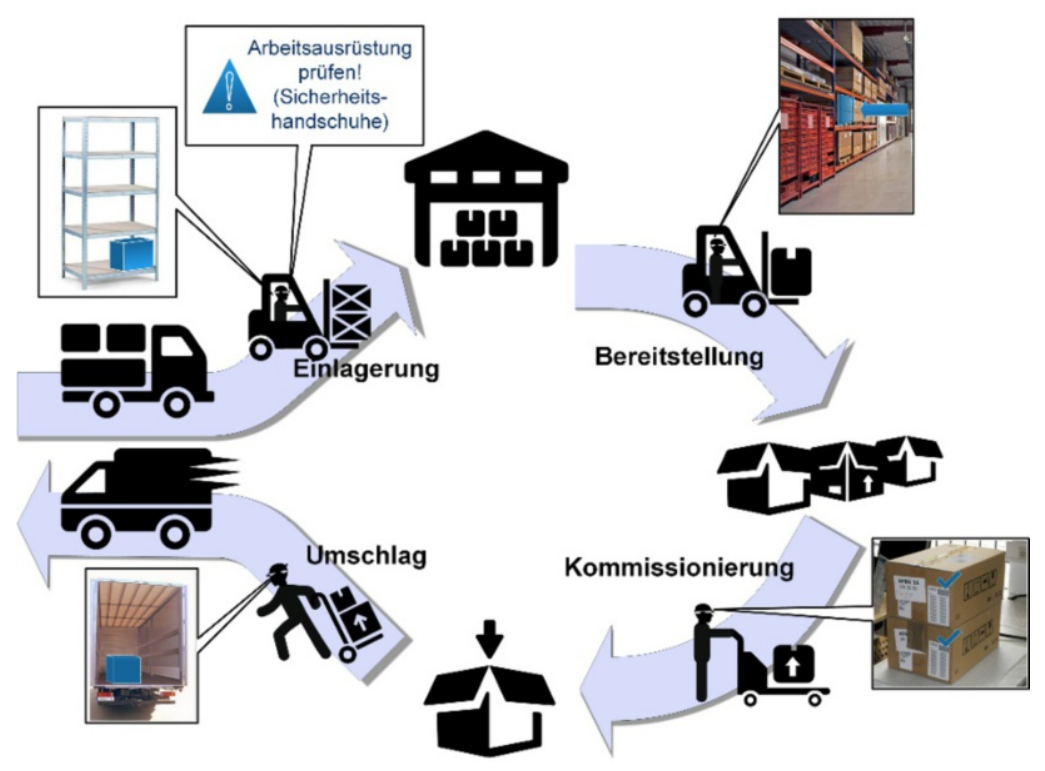

Abb. 1. Unterstützung der Logistikwertschöpfungskette durch GLASSHOUSE

Die Logistikwertschöpfungskette ist geprägt durch die Hauptbereiche Einlagerung, Bereitstellung, Kommissionierung sowie Umschlag (vgl. Abb. 1). Bereits in der Einlagerung können dem Lagerarbeiter wertvolle Informationen mit Hilfe von Smart Glasses angezeigt werden. Unterstützende Informationen können die exakte Positionierung und Stapelung des Stückguts sein. Weiterhin können Warn- und 
Sicherheitshinweise eingeblendet werden, bspw. bei zerbrechlicher Ware. Auch Informationen hinsichtlich eines optimalen Pufferplatzes können den gesamten Warenfluss im Lager verbessern. Eine Navigation hilft dem Lagermitarbeiter beim Prozess der Bereitstellung, um sich anhand von eingeblendeten Pfeilen in einem großen Lager besser zu orientieren. In der Kommissionierung ermöglicht die visuelle Darstellung mit Smart Glasses einen unterbrechungsfreien Arbeitsprozess. Da der Lagermitarbeiter beide Hände frei hat, werden Phasen zwischen Tragen des Stückguts und Arbeitsunterbrechungen durch die Verwendung eines klassischen MDE-Scanners oder papierbasierter Picking-Listen gänzlich vermieden. Zudem findet kein Medienbruch statt wie es bei papierbasierten Picking-Listen vorkommt, der Status des Kommissionierungsprozesses kann direkt in einem Lagerhaltungssystem gespeichert werden. Beim Umschlagsprozess kann identisch zur Lagerung eine Visualisierung des Lagerplatzes erfolgen, um so eine optimale Auslastung und Beladung zu erzielen.

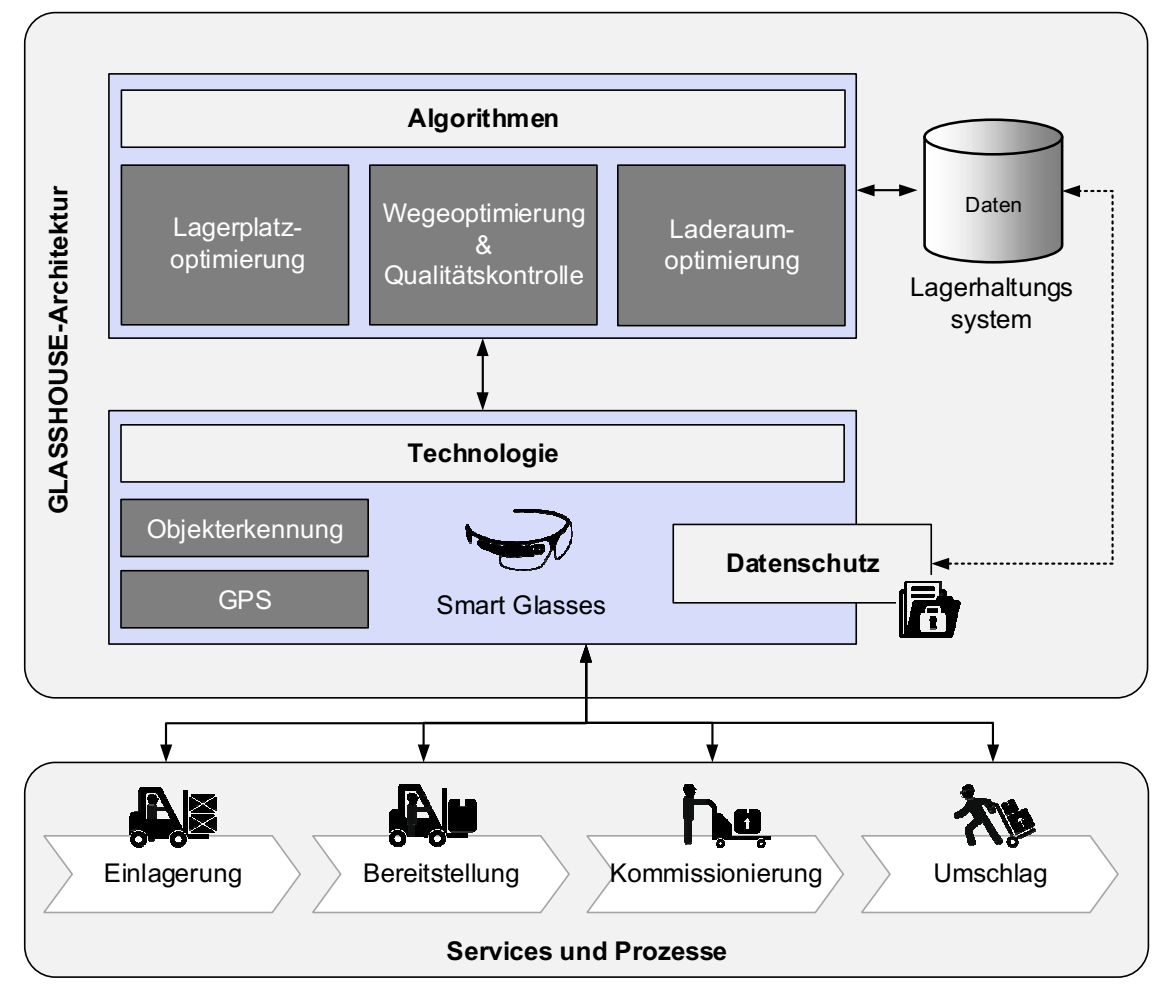

Abb. 2. GLASSHOUSE-Architektur

Eine zentrale Architektur ist fundamental notwendig, um eine durchgängige Informationsversorgung in allen Wertschöpfungsprozessen von der Einlagerung bis hin zum Umschlag zu gewährleisten (vgl. Abb. 2). Dabei steht eine einheitliche 
Benutzeroberfläche für alle Bereiche im Vordergrund, welche verständlich und intuitiv ist, um die Akzeptanz für die Technologie zu erhöhen und die Einlernphase in das System so gering wie möglich zu halten. Die technologischen Funktionalitäten wie Objekterkennung oder GPS der Smart Glasses lassen weitere nutzenstiftende Anwendungsfälle erwarten. Aufgrund der in Blickhöhe befestigten Kamera und der Verarbeitung der Sensordaten von Smart Glasses ist der Datenschutz ein zu beachtender negativer Aspekt. Um eine von den Endnutzern akzeptierte Anwendung zu erzielen, muss diesem Kritikpunkt wirkungsvoll begegnet werden. Daher sieht das Projekt GLASSHOUSE eine umfassende datenschutzrechtliche Betrachtung der von Smart Glasses erfassten und weiterverarbeiteten Daten vor.

\subsection{Technische Ziele}

Damit die Unterstützungspotenziale von Smart Glasses entlang aller Wertschöpfungsprozesse der Logistik erreicht werden, ist ein integratives Systemkonzept aller Anwendungsfälle bzw. logistischen Prozesse unter Berücksichtigung der technischen Eigenschaften von Smart Glasses notwendig.

Daher verfolgt GLASSHOUSE als Ziel die Konzeption eines ganzheitlichen Systemkonzepts, welches sämtliche Wertschöpfungsprozesse und Anwendungsfälle in der Logistik mit einem Smart-Glasses-basierten Assistenzsystems unterstützt. Da die datengetriebenen Logistikprozesse stark auf Entscheidungen und Berechnungen aufbauen, die stetig aktualisiert werden, ist ein cloud-basierter Ansatz notwendig. In der Cloud können rechenintensive Operationen durchgeführt werden. Durch eine Bereitstellung von Schnittstellen können Informationen mit dem Smart-Glasses-basierten System ausgetauscht werden. Durch diesen Ansatz wird ein Nachteil von Smart Glasses, die limitierte Datenverarbeitungskapazität, aufgehoben.

Ein konkreter Anwendungsfall für eine Smart-Glasses-basierte Unterstützung eines Prozesses ist der Zusammenbau und die Bestückung von Werbedisplays für den Einzelhandel, die in der Intralogistik im Rahmen sog. Mehrwertdienstleistungen übernommen werden (vgl. Abb. 3). Der Nutzer erhält eine kontextsensitive Prozessführung eingeblendet, die visuelle Hinweise zu jedem Prozessschritt mittels Text und Bildern enthält. Die Schnittstelle zur Datenhaltung in der Cloud ermöglicht die Bereitstellung von adaptiven Stücklisten, somit wird ein dynamischer Aufbau der Anwendung ermöglicht und eine kontinuierliche Möglichkeit zur Aktualisierung wird erreicht. Diese beispielhafte Anwendung zeigt den Bedarf für ein ganzheitliches Systemkonzept mit einem integrierten cloud-basierten Ansatz auf, um die Nutzenpotenziale von Smart Glasses ausnahmslos in allen logistischen Prozessen zu erreichen. 


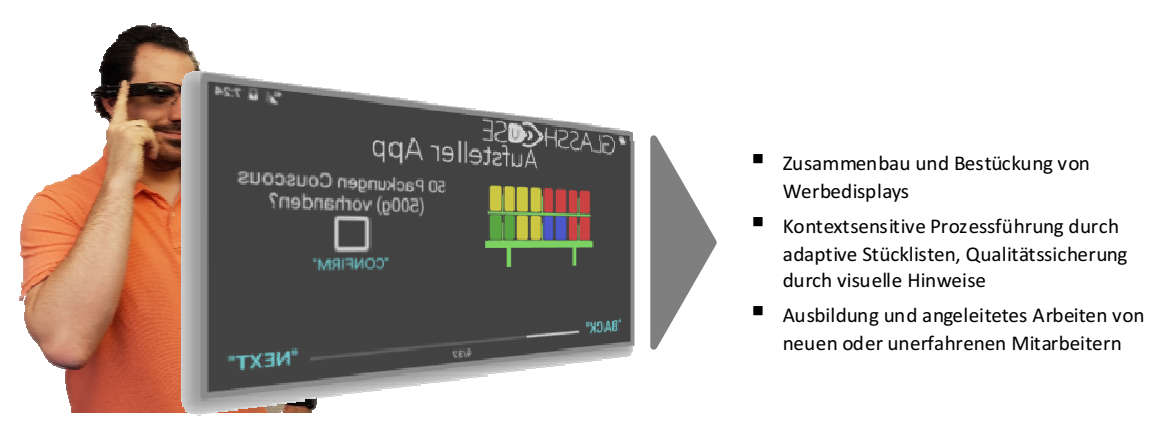

Abb. 3. Smart-Glasses-Anwendung in der Intralogistik und im Einzelhandel

\subsubsection{Konzeption eines ganzheitlichen Logistiksystems}

Die Konzeption des ganzheitlichen Logistiksystems zielt auf ein akzeptiertes und anwenderfreundliches Design des Systems, welches unter Berücksichtigung der Anwendungsfälle ein möglichst breites Spektrum an logistischen Prozessen unterstützt.

Anfangs erfolgt eine Anforderungserhebung, hierfür wird der State-of-the-Art an Logistikunterstützungssystemen erhoben und verglichen. Im Fokus steht vorrangig der Vergleich der Wirtschaftlichkeit und Anwendbarkeit. Daher werden objektive Kriterien zur Bewertung verschiedener Unterstützungssysteme aufgestellt und für den Vergleich herangezogen. Besonders wird der Prozess der Kommissionierung für den Vergleich ausgewählt, da in der Praxis verschiedenste Methoden der Kommissionierung existieren, wie Pick-by-Paper, Pick-by-Scan, Pick-by-Voice, Pick-by-Light und die Smart-Glasses-basierte Methode Pick-by-Vision.

Die zu unterstützenden Logistikprozesse benötigen verschiedene Teilsysteme und Module, sowohl ausgelagert in der Cloud als auch direkt betrieben auf der Smart Glasses. Eine zu konzipierende Systemübersicht und entwickelte Referenzarchitektur unterstützt bei der Entwicklung von Smart-Glasses-basierten Systemen in der Logistik, um einerseits eine Konsolidierung verschiedenster Teilsysteme vorzunehmen und um andererseits eine klare Abgrenzung vornehmen zu können. Schließlich ermöglicht eine Referenzarchitektur die Festlegung von notwendigen Schnittstellen zwischen Teilmodulen sowie eine Reduktion von redundanten Implementierungen. Im engen Zusammenhang mit festgelegten Teilmodulen steht die technische Ausgestaltung zur Berücksichtigung des Datenschutzes. Ein Vorschlag zur Realisierung des Privacy-by-Design- bzw. Privacy-by-DefaultAnsatzes innerhalb Smart-Glasses-basierter Systeme ist erforderlich, um die Datenschutzgrundverordnung und betriebsrechtliche Anforderungen zu erfüllen.

Schließlich erfolgt anknüpfend die Simulation und Planung von Lager- und Materialflusssystemen, um die Potenziale sowie die Effizienzen vorhandener logistischer Algorithmen zur Lagerplatzoptimierung, Wegefindung durch Läger und Laderaumoptimierungen und deren möglichen Integration in das Smart-Glassesbasierte System zu bewerten. Ein Konglomerat von verschiedenen logistischen Al- 
gorithmen bildet dabei den Kern der intelligenten Prozesssteuerung. Hier werden Optimierungen in Echtzeit durchgeführt, die den Benutzer dazu befähigen, seine Tätigkeit besser durchführen zu können. Um dies erreichen zu können, muss konzeptionell erarbeitet werden, welche Daten, Algorithmen und Systeme benötigt werden, damit auf den Smart Glasses die richtigen Informationen angezeigt werden können. So können bspw. die kürzesten Wege für den jeweiligen Benutzer oder beim Packen von LKW die beste Packreihenfolge und Lage der Güter berechnet werden.

\subsubsection{Cloud-basierter Ansatz}

Das im Projekt GLASSHOUSE zu entwickelnde Smart-Glasses-basierte System sieht die Unterstützung der Logistikprozesse vor. Dabei sollen mittels Smart Glasses die Arbeiter durch die Prozesse geführt werden. Die Realisierung der Architektur ermöglicht es, den geringen Speicherkapazitäten sowie Rechenleistungen von Smart Glasses effektiv zu begegnen (vgl. Abschnitt 3). Die Smart Glasses teilen der Cloud mit, in welchem Prozessschritt sie sich aktuell „befinden“, und übermitteln gegebenenfalls Parameter, die zur Ausführung der Services benötigt werden. Die Cloud verarbeitet diese Anfragen und definiert eine individuelle Lösung, welche dann dem Nutzer per Smart Glasses wieder bereitgestellt wird. Das technische Arbeitsziel ist somit die Implementierung eines integrierten, cloudbasierten Demonstrators. Ein wissenschaftliches Teilarbeitsziel ist dabei die nutzerfreundliche Gestaltung und Akzeptanz des Systems, die einhergeht mit den festgelegten Forschungs- sowie Anwendungszielen.

\subsection{Forschungsziele}

Forschungsziel von GLASSHOUSE ist die Erhebung von Anwendungsfällen von Smart Glasses innerhalb der logistischen Prozesse, die Erhebung der Informationsund Unterstützungsbedarfe der Mitarbeiterinnen und Mitarbeiter sowie die Systemgestaltung unter Berücksichtigung der Akzeptanz und des Datenschutzes der Anwender.

Hierfür ist es notwendig, im Rahmen einer Anforderungsanalyse die Prozesse der Logistikwertschöpfungskette aufzunehmen und den jeweiligen Informationsbedarf in den einzelnen Prozessabschnitten zu analysieren. Benötigte Informationen sollen durch das Smart-Glasses-basierte Assistenzsystem zur Verfügung gestellt werden. Dabei befasst sich die Anforderungsanalyse explizit mit der Nutzung des Systems aus Anwendersicht und dient dazu, die Erwartungen der zukünftigen Nutzerinnen und Nutzer an die Smart-Glasses-Unterstützung abzubilden. Zur Erhebung der Anforderungen werden Prozessbeobachtungen (engl:: shadowing) und Experteninterviews mit Mitarbeiterinnen und Mitarbeitern aus den involvierten Wertschöpfungsbereichen durchgeführt. Eine anschließende Fokusgruppe mit allen Stakeholdern aus dem Verbundprojekt bewertet die verschiedenen Anwendungsfälle nach ihrem Nutzen und dem technischen Aufwand für ihre 
Realisierung, sodass frühzeitig besonders nutzenbringende Anwendungsfälle identifiziert werden. Unter Berücksichtigung der ersten Erkenntnisse findet eine Technologieauswahl der zu verwendenden Smart Glasses im Projekt statt. Entscheidende Auswahlkriterien sind Faktoren zur Gebrauchstauglichkeit wie Ergonomie, Tragekomfort, Ausdauer der Akkuleistung, Auflösung des Displays und Interaktionsmöglichkeiten.

In Bezug auf das Datenschutzkonzept werden die rechtlichen Rahmenbedingungen sowie arbeitsrechtliche Aspekte und Akzeptanzbarrieren untersucht. Das zu entwickelnde Smart-Glasses-basierte Assistenzsystem wird - insb. im Hinblick auf die ursprünglichen Anforderungen - im praxisnahen Einsatz umfangreich evaluiert. Durch iterative Design- und Evaluationszyklen wird die Systemgestaltung kontinuierlich verbessert. Insb. für Smart-Glasses-basierte Systeme existieren bisher wenig bis kaum Designprinzipien und Interaktionskonzepte für die Gestaltung entsprechender Systeme. Daher ist ein weiteres Forschungsziel im Projekt, diese Prinzipien basierend auf der Rückmeldung der Anwender aufzustellen und kontinuierlich durch neue Technologien anzupassen und zu erheben.

\subsection{Anwendungsziele}

Oberstes Anwendungsziel des Projekts ist die Erprobung und Evaluation der Anwendbarkeit des entwickelten Smart-Glasses-basierten Systems in der Logistik. Dafür wird das System sowohl bei Hellmann in der Kontraktlogistik und im Crossdock als auch bei Meyer \& Meyer in der Fashionlogistik erprobt. Im Fokus der Untersuchung stehen die technische Infrastruktur und Schnittstellen der Logistiksysteme, um die Kommunikation der GLASSHOUSE-Architektur (vgl. Abb. 2) zu spezifizieren. Ferner werden datenschutzrechtliche Rahmenbedingungen definiert. Zudem soll die Interaktion mit Smart Glasses aus Sicht des Anwenders analysiert und aktiv mitgestaltet werden, um eine intuitive Form der Informationsbereitstellung unter Verwendung innovativer Technologien zu entwickeln. Weiterhin sind folgende Anwendungsziele für eine akzeptierte und wirtschaftliche Lösung entscheidend:

- Abdeckung der Wertschöpfungskette von Einlagerung, Bereitstellung, Kommissionierung und Umschlag durch das System,

- Steigerung der Prozessqualität und höhere Produktivität über die Wertschöpfungskette hinweg durch weniger Fehler, weniger Handgriffe, Verringerung von Wegen und Automatisierung von Tätigkeiten (z. B. Scannen oder Vermessen von Packstücken, etc.) sowie Erhöhung der Mitarbeitersicherheit und -zufriedenheit,

- Einsatz kostengünstiger Technologien und geringe Einstiegshürden und

- Unterstützung der Mitarbeiterinnen und Mitarbeiter und die Schaffung eines attraktiven Arbeitsplatzes. 
Die Anwendungsziele werden im Sinne der Konsortialforschung in Zusammenarbeit zwischen Forschung und Praxis erreicht. Das Projektkonsortium GLASSHOUSE besteht zum einen aus den wissenschaftlichen Partnern: Fachgebiet Informationsmanagement und Wirtschaftsinformatik (IMWI) der Universität Osnabrück sowie dem Fachgebiet Betriebswirtschaftslehre, insb. Logistikmanagement der Hochschule Osnabrück. Zum anderen sind die Anwendungspartner Hellmann Worldwide Logistics SE \& Co. KG sowie Meyer \& Meyer Logistics SE \& Co. KG und der Implementierungspartner pco Personal Computer Organisation GmbH \& Co. KG mit am Projektvorhaben beteiligt.

Die Hellmann Worldwide Logistics SE \& Co. KG (Hellmann) ist ein deutsches Transport- und Logistikunternehmen mit Sitz in Osnabrück. Als global agierender Logistikdienstleister ist Hellmann mit einem weltweiten Netzwerk von 10.696 Mitarbeiterinnen und Mitarbeitern in 257 Niederlassungen in 56 Ländern vertreten und erwirtschaftet dabei einen Umsatz von 2,54 Mrd. €. Hellmann ist tätig in den Bereichen Logistikdienstleistungen (u.a. Automotive, Fashion, Perishable Goods und Hi-Tech), Warehousing, Gütertransport auf der Straße, Schiene, per Schiff und Flugzeug, IT-Lösungen, Consulting, Entsorgungsdienstleistung und Supply Chain Management. Mit GLASSHOUSE sollen zum einen die Mitarbeiter durch mobile Smart Glasses ergonomisch entlastet und vor Arbeitssicherheitsfehlern geschützt werden, zum anderen sollen Qualität und Schnelligkeit der Prozesse über die gesamte Wertschöpfungskette gesteigert werden.

Das Osnabrücker Familienunternehmen Meyer \& Meyer ist ein erfahrener Partner der Fashionbranche und begleitet seine Kunden entlang der gesamten Supply Chain - „from sheep to shop“. Als führender Spezialist für Fashionlogistik in Europa bietet das Unternehmen ein Leistungsspektrum, das von der Rohwarenund Produktionslogistik über die Lagerung, Aufbereitung und Qualitätssicherung bis zur verkaufsfertigen Distribution der Waren in den Einzelhandel oder an den Endkunden reicht. Meyer \& Meyer steuert und verknüpft Wertschöpfungsketten mit Hilfe modernster IT-Technologien und bietet gemeinsam mit Partnern intelligente Omnichannel-Lösungen. Neben der Fashionlogistik hat Meyer \& Meyer weitere spezifische Logistiklösungen für Automotive und diverse besonders sensible Güter entwickelt. Aus der Firmenzentrale sowie einem Netz von Niederlassungen und Partnergesellschaften im In- und Ausland steuert Meyer \& Meyer mit 1.800 Mitarbeitern logistische Aktivitäten in Europa, Asien und Nordafrika. Das Interesse an dem Projekt begründet sich in der Technikaffinität und der Chance den Mitarbeiterinnen und Mitarbeitern einen attraktiven und ergonomischen Arbeitsplatz zu schaffen.

Das 1984 gegründete Unternehmen pco Personal Computer Organisation $\mathrm{GmbH} \& \mathrm{Co}$. KG (PCO) hat fundierte Erfahrung im Bereich der Begleitung von IT-Systemen in der Logistik. Mit über 100 Mitarbeiterinnen und Mitarbeitern, etwa 26 Mio. $€$ Umsatz und Projekten in über 40 Standorten hat sich das ursprüngliche Systemhaus inzwischen zu einem Anbieter komplexer IT-Dienstleistungen entwickelt und bedient ein differenziertes Feld von Kunden aus allen Branchen. Für das Projekt bringt PCO seine über viele Jahre ausgeprägte Projekterfahrung 
und verschiedene Technologiekompetenzen, insb. in den Bereichen Mobilität, Infrastruktur und Service Management, im speziellen Logistikdienstleistungen, ein, weshalb der vorliegende integrierte Ansatz über die gesamte Wertschöpfungskette hinweg für PCO ein ideales Betätigungsfeld darstellt. Dabei liegt das besondere Interesse in einer wirtschaftlichen Ausrichtung des Projekts und in der Möglichkeit, zukünftige Technologien in der Logistik federführend mitgestalten zu können.

Weiterhin war neben den oben genannten Projektpartnern am Forschungsprojekt GLASSHOUSE als assoziierter Partner das Kompetenznetz Individuallogistik e.V. (KNI) beteiligt, das bei der Öffentlichkeitsarbeit und dem Transfer der erzielten Projektergebnisse unterstützend mitgewirkt hat.

\section{Umfeldanalyse und Abgrenzung}

\subsection{Stand der Technik und Technologieauswahl}

Ein abgestimmter Auswahlprozess unter allen Stakeholdern und die Herleitung von Entscheidungskriterien zur Auswahl und Priorisierung geeigneter Hardware ist insb. bei neuartigen Technologien entscheidend für das Gelingen eines Projektes. Relevante Entscheidungskriterien für eine Technologieauswahl sind u.a. die Akkulaufzeit, Auflösung des Displays, Tragekonform sowie Interaktionsmöglichkeiten (vgl. Tabelle 1).

Tabelle 1. Auszug relevanter Entscheidungskriterien für die Technologieauswahl

\begin{tabular}{ll}
\hline Anforderung & \multicolumn{1}{c}{ Beschreibung } \\
\hline $\begin{array}{l}\text { Industrie- } \\
\text { tauglichkeit }\end{array}$ & $\begin{array}{l}\text { Da Smart Glasses anfänglich für den Konsumentenmarkt entwickelt } \\
\text { wurden, sind nicht alle Smart Glasses für den industriellen Einsatz ge- } \\
\text { eignet. Daher sollte auf eine robuste Fertigung geachtet werden. }\end{array}$ \\
$\begin{array}{l}\text { Ergonomie und } \\
\text { Tragekonform } \\
\text { Eine gute Ergonomie und Tragekonform sind für die Akzeptanz der Nut- } \\
\text { zerinnen und Nutzer entscheidend, insb. wenn der Einsatz der Smart } \\
\text { Glasses über eine gesamte Arbeitsschicht erfolgen soll. }\end{array}$ \\
$\begin{array}{l}\text { Auflösung und } \\
\text { Positionierung Auflösung des Displays ist wichtig, damit detaillierte Informationen } \\
\text { des Displays }\end{array}$ & $\begin{array}{l}\text { ohne Anstrengungen von den Anwenderinnen und Anwendern gelesen } \\
\text { werden können. Eine variable Positionierung des Displays ermöglicht es }\end{array}$ \\
ihnen, bei monokularen Brillen das Display auf die Seite des dominanten \\
Auges zu positionieren.
\end{tabular}


Die Auswahl einer geeigneten Smart Glasses fand im GLASSHOUSE-Projekt detailliert statt. Nach einem umfangreichen Technologieauswahlprozess aus einer Menge von 20 Smart Glasses fand eine Eingrenzung auf fünf Smart-Glasses-Modelle statt (vgl. Tabelle 2). Zu den wichtigsten Herstellern zählen Vuzix, Google, Epson und ODG-Design-Group.

Tabelle 2. Vergleich der technischen Eigenschaften

\begin{tabular}{|c|c|c|c|c|c|}
\hline Merkmal & $\begin{array}{l}\text { Vuzix } \\
\text { M100 }\end{array}$ & $\begin{array}{l}\text { Vuzix } \\
\text { M300 }\end{array}$ & $\begin{array}{l}\text { Google } \\
\text { Glass }\end{array}$ & $\begin{array}{l}\text { Epson } \\
\text { Moverio } \\
\text { BT-200 }\end{array}$ & ODG R7 \\
\hline Displayart & $\begin{array}{l}\text { VGA- } \\
\text { Display }\end{array}$ & $\begin{array}{l}\text { LCD- } \\
\text { Display }\end{array}$ & Prisma & Prisma & Prisma \\
\hline Displayauflösung & $432 \times 240$ & $640 \times 360$ & $640 \times 360$ & $960 \times 540$ & $720 p$ \\
\hline $\begin{array}{l}\text { Position des } \\
\text { Displays }\end{array}$ & anpassbar & anpassbar & nur rechts & binokular & binokular \\
\hline Kamera & $5 \mathrm{MP}$ & $10 \mathrm{MP}$ & $5 \mathrm{MP}$ & $5 \mathrm{MP}$ & $1080 p$ \\
\hline Verbindung & $\begin{array}{c}\text { Wi-Fi; } \\
\text { Bluetooth } \\
\text { 4.0: Micro- } \\
\text { USB }\end{array}$ & $\begin{array}{l}\text { Wi-Fi; } \\
\text { Bluetooth } \\
\text { 4.1: Micro- } \\
\text { USB }\end{array}$ & $\begin{array}{c}\text { UMTS; } \\
\text { Wi-Fi; } \\
\text { Bluetooth; } \\
\text { Micro-USB; } \\
\text { GPS }\end{array}$ & $\begin{array}{l}\text { Wi-Fi, } \\
\text { Bluetooth } \\
3.0\end{array}$ & $\begin{array}{c}\text { Wi-Fi; } \\
\text { Bluetooth } \\
\text { 4.1; GPS }\end{array}$ \\
\hline Laufzeit & $1-6 \mathrm{~h}$ & $2-12 \mathrm{~h}$ & $1 \mathrm{~h}$ & $6 \mathrm{~h}$ & $\begin{array}{l}\text { keine } \\
\text { Angabe }\end{array}$ \\
\hline $\begin{array}{l}\text { Interaktions- } \\
\text { möglichkeiten }\end{array}$ & $\begin{array}{l}\text { Knopf- } \\
\text { steuerung; } \\
\text { Touchpad; } \\
\text { Sprach- } \\
\text { steuerung }\end{array}$ & $\begin{array}{l}\text { Knopf- } \\
\text { steuerung; } \\
\text { Touchpad; } \\
\text { Sprach- } \\
\text { steuerung }\end{array}$ & $\begin{array}{l}\text { Knopf- } \\
\text { steuerung; } \\
\text { Touchpad; } \\
\text { Sprach- } \\
\text { steuerung }\end{array}$ & Controller & $\begin{array}{c}\text { Touchpad; } \\
\text { Knöpfe }\end{array}$ \\
\hline Datenhaltung & $\begin{array}{c}4 \mathrm{~GB}+32 \\
\text { GB SD- } \\
\text { Karte }\end{array}$ & $64 \mathrm{~GB}$ & $16 \mathrm{~GB}$ & $\begin{array}{c}8 \mathrm{~GB}+32 \\
\text { GB SD- } \\
\text { Karte }\end{array}$ & $64 \mathrm{~GB}$ \\
\hline Betriebssystem & Android 4.4 & Android 6.0 & $\begin{array}{c}\text { Android } \\
4.4 .2\end{array}$ & $\begin{array}{c}\text { Android } \\
4.0 .4\end{array}$ & $\begin{array}{c}\text { angepasstes } \\
\text { Android }\end{array}$ \\
\hline Tragekomfort & $\begin{array}{l}\text { niedrig - } \\
\text { einseitige } \\
\text { Gewichts- } \\
\text { belastung }\end{array}$ & $\begin{array}{l}\text { gut - Ge- } \\
\text { wichts- } \\
\text { verteilung }\end{array}$ & $\begin{array}{l}\text { gut - gerin- } \\
\text { ges Gewicht }\end{array}$ & $\begin{array}{l}\text { gut - Ge- } \\
\text { wichts- } \\
\text { verteilung }\end{array}$ & $\begin{array}{l}\text { gut - Ge- } \\
\text { wichts- } \\
\text { verteilung }\end{array}$ \\
\hline $\begin{array}{l}\text { Industrie- } \\
\text { tauglichkeit }\end{array}$ & gegeben & gegeben & $\begin{array}{l}\text { nicht } \\
\text { gegeben }\end{array}$ & gegeben & gegeben \\
\hline Preis & $999 €$ & $1.499 €$ & $1.499 €$ & $699 €$ & $2.750 €$ \\
\hline
\end{tabular}

Basierend auf den herangezogenen Entscheidungskriterien und den technologischen Eigenschaften hat sich das Projektkonsortium für die Vuzix M300 entschieden (zu einer ausführlichen Erläuterung der Smart Glasses vgl. S. 159). Dabei erfolgte die Abwägung durch hervorgehobene technologische Eigenschaften, die für einen erfolgreichen Einsatz in der Intralogistik notwendig sind. Die Vuzix M100 
ist in allen technologischen Aspekten dem Nachfolger der Vuzix M300 unterlegen und wurde daher nicht weiter betrachtet. Primär wurde die Google Glass aufgrund ihrer schlechten Verfügbarkeit und Einstellung des Supportes nicht ausgewählt. Zudem kam das Konsortium in ersten Tests zu der Bewertung, dass die industrielle Tauglichkeit durch das ungeschützte Glasprisma nicht gegeben ist. Obwohl die Epson Moverio den technologischen Eigenschaften der Vuzix M300 ähnelt und ein binokulares Display besitzt, wurde diese Smart Glasses aufgrund der ausschließlichen Steuerung über einen Controller ausgeschlossen. Der positive Effekt einer Smart Glasses der handfreien Steuerung wurde durch den Einsatz eines Controllers erheblich reduziert. Die ODG R7 war zum Betrachtungszeitpunkt nur in den USA verfügbar. Ferner verwendet die Brille ein stark modifiziertes AndroidBetriebssystem, wodurch die Übertragbarkeit auf andere Smart-Glasses-Modelle nicht gegeben ist. Zudem kann die Epson Moverio und die ODG R7 nicht mit einer normalen Brille zusammengetragen werden, wodurch eine große Nutzergruppe ausgeschlossen wird.

Positive wurde vom Konsortium insb. der schnelle Austausch des Akkus sowie die Erweiterungsmöglichkeiten zur Verbesserung des Tragekomforts der Vuzix M300 bewertet. Schließlich ermöglicht eine neue Version des Betriebssystems Android die gewünschte, individuelle Entwicklung der Smart-Glasses-basierten Anwendungen.

\subsection{Stand der Praxis}

Im Gegensatz zum Konsumentenmarkt konnten Smart-Glasses-Hersteller ihre Modelle besser im betrieblichen Umfeld vertreiben. Waren früher die Technologien hardwaretechnisch zu unausgereift für einen betrieblichen Einsatz oder zu teuer, ermöglichen neuere Modelle einen nutzenstiftenden Einsatz innerhalb betrieblicher Prozesse. Neue Erwartungen an die Technologie beruhen insb. auf eine ausgereiftere Hardware der Hersteller mit praxistauglicheren Eigenschaften.

Entsprechende Erwartungen werden insb. von namenhaften IT-Unternehmen geschürt, wie Google mit dem Modell Google Glass. Mittlerweile haben viele groBe Firmen, aber auch Technologie-Startups, verschiedene Smart Glasses entwickelt, sodass bspw. Produkte von Epson (2019) oder Vuzix (2019) verfügbar sind. Weiterhin wird die Forschung und Entwicklung im Bereich Augmented Reality von Unternehmen wie Apple (Ma et al. 2019) und Microsoft (2019) vorangetrieben und münden in neue Patentanmeldungen.

Mitarbeiter und Mitarbeiterinnen ausgestattet mit einer Smart Glasses machen weniger Fehler beim Picking-Prozess und benötigen weniger Zeit, um kommissionierende Pakete zu finden (DHL Trend Research 2014). Damit wird die Pick-byVision-Anwendung zu dem Hauptanwendungsfall für Smart Glasses in der Logistik. Beim betrieblichen Einsatz steht die Logistikbranche vor der Herausforderung abzuschätzen, inwiefern eine Smart-Glasses-basierte Anwendung welchen technischen und softwareseitigen Anforderungen genügen muss, damit eine Praxistaug- 
lichkeit und Akzeptanz der Anwender gewährleitet ist. Diese Faktoren sind u.a. eine Grundvoraussetzung, um erhoffte Prozessverbesserungen zu erzielen.

Aufgrund der frühzeitigen Identifikation der Vorteilhaftigkeit von Smart Glasses im Kommissionierprozess haben Logistiker und IT-Unternehmen erste betriebliche Lösungen für diesen speziellen Teilprozess entwickelt.

SAP (2014) entwickelte den AR Warehouse Picker, dessen Anwendung auf verschiedensten Android-basierten Smart Glasses funktionsfähig ist. Durch die direkte Verknüpfung mit ihrem ERP-System kann der Kommissionierer direkt die nächsten Aufträge abrufen und wird durch den Prozess Schritt für Schritt geführt. Auch der Anbieter von Intralogistiksystemen Knapp AG entwickelte eine Pick-byVision-Lösung, die den Kommissionierer unterstützt. Weitere initialisierte Projekte zwischen IT-Dienstleistern und Logistikern resultierten in ähnlichen Systemlösungen. Pcdata (2014) stellt mit ihrer Pick-by-Vision-Lösung eine Effizienzsteigerung von 20-50 \% gegenüber dem Pick-by-Paper-Ansatz fest. Die Lösung wurde für die Vuzix M100 Smart Glasses entwickelt. Auffallend bei der Betrachtung des Standes der Praxis ist das bisherig eine Konzentration auf Pick-by-Vision-Lösungen existieren. Eine gesamtheitliche Smart-Glasses-basierte-Lösung zur Unterstützung von Logistikprozessen von der Lagerung über Kommissionierung bis hin zum Umschlag fehlt bisherig und ist daher Gegenstand des GLASSHOUSE-Projekts.

\subsection{Stand der Wissenschaft}

Betrachtet man den Bereich der Wissenschaft, so ist der Begriff „,erweiterte Realität“ 1992 durch Caudell und Mizell (1992) begründet worden. Davor gab es bereits Entwicklungen unter dem Namen Head-Mounted Display (HMD), welche bis 1968 zurückgehen (Sutherland 1968). Eine erstmalige nennenswerte Verbreitung von Datenbrillen bzw. Smart Glasses fand mit der Google Glass statt. Als wesentliche Mehrwerte von HMD bei der Prozessunterstützung, bspw. in der Wartung und Instandhaltung, wurden das schnellere Auffinden und Abarbeiten von Aufgaben bzw. Fehlern am Gerät sowie eine Reduzierung der Kopf- und Nackenbewegungen im Gegensatz zur Nutzung von Handgeräten und somit körperliche Belastungen festgestellt (Henderson und Feiner 2011). Darüber hinaus ist aktuell die Mensch-Maschine-Schnittstelle in Form der Interaktion zwischen Nutzer und ARSystem, aber auch die Entwicklung einer realistischen Einbettung von Information in die Umwelt relevant (Meier et al. 2011). Aufgrund der neuentwickelten günstigeren Geräte stieg die Relevanz, sodass in den letzten Jahren vermehrt die Nutzung von AR in verschiedenen Anwendungsfeldern diskutiert wird. Hierzu gehört auch die Logistikbranche. An der TU München bspw. wurde in dem vom BMWi geförderten Projekt Pick-by-Vision (AiF-FV-Nr. 14756 N) die benutzeroptimale Informationsbereitstellung mit Hilfe einer durch den Kommissionierer zu tragenden Datenbrille, in der in Abhängigkeit von Ort, Zeit, Blickfeld sowie dem Stand der Auftragsbearbeitung alle relevanten Daten zur fehlerfreien Durchführung der Kommissionierungsaufgabe untersucht (Günthner et al. 2009). Ein weiteres For- 
schungsvorhaben verglich eine papierbasierte Kommissionierung mit Pick-ByVoice (stimmgeleitetes Verfahren), AR-basiertes Pick-By-Voice-System und eine VR-Simulation zum Kommissionierungsprozess, wobei im Ergebnis die Systeme eine hohe Akzeptanz der Nutzer erreichten und die Motivation förderten. Nachteile waren die damals schlechte Spracherkennung der genutzten Hardware (Reif und Walch 2008). Auch in diesem Forschungsvorhaben wurde lediglich der Kommissionierungsprozess betrachtet.

Genau an dieser Stelle setzt GLASSHOUSE an, um die Forschungslücke der vollständigen Abdeckung der gesamten Wertschöpfungskette in der Logistik zu schließen und die Einzelforschungen zum Bereich Kommissionierung in eine Gesamtlösung zur digitalen Dienstleistungsunterstützung zusammenzufassen. Diese Forschungslücke bestätigt sich bei der Betrachtung des aktuellen Stands der Praxis sowie der Wissenschaft.

\section{Zusammenfassung und Ausblick}

Mit GLASSHOUSE werden aktuelle Herausforderungen in der Logistik effektiv durch den Einsatz von Smart Glasses entlang der gesamten Wertschöpfungskette begegnet. Die entwickelte Smart-Glasses-basierte Systemlösung unterstützt in den Prozessen Einlagerung, Bereitstellung, Kommissionierung und Umschlag.

Dabei zielt die Lösung auf eine Verkürzung der Einlernphasen in den logistischen Prozessen ab, um der saisonbedingten Mitarbeiterfluktuation zu begegnen. Prozessabläufe können durch die Durchführung von bimanuellen Tätigkeiten verbessert werden. Insb. hierfür erforschte und bewertete das Konsortium umfangreiche Einsatzszenarien. Dabei wurde stets die Gestaltung eines attraktiven und ergonomischen Arbeitsplatzes fokussiert und Anwender wurden mit in den Gestaltungsprozess einbezogen. Bei der Systementwicklung wurden Faktoren wie Gebrauchstauglichkeit, Akzeptanz, Datenschutz, Wirtschaftlichkeit, Ergonomie, Systemdesign und der Architekturaufbau der Lösung berücksichtigt. Hieraus entstanden IT-Artefakte wie ein Use-Case-Katalog, eine Referenzarchitektur für den modularen Aufbau von Smart-Glasses-basierten Systemen, eine Cloud-Infrastruktur sowie eine Low-Code-Plattform. Ferner wurde eine „Best-Practice-Vorgehenswei-

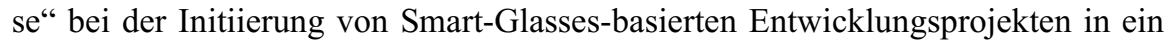
Rahmenwerk modelliert. Zusätzlich hergeleitete Designprinzipien geben Empfehlungen bei der Gestaltung von Smart-Glasses-basierten Anwendungen.

Das Projekt GLASSHOUSE zeigt auf, welche Überlegungen bei der Systemgestaltung von neuen Technologien innerhalb von Arbeitsprozessen vorgenommen werden müssen, um eine nutzenstiftende und akzeptierte Lösung zu erzielen. Dabei können die gewonnenen Erkenntnisse wie Designprinzipien oder auch Vorgehensweisen auf andere Branchen und Industrien übertragen werden. Die Systemgestaltung erfolgte konsequent von der Identifikation erster Anwendungsfälle bis hin zur Priorisierung von ersten fachkonzeptionellen Entwürfen sowie deren Um- 
setzung und Evaluation. Ein Erfolgsfaktor war der stetige Einbezug verschiedener Stakeholder, um differenzierte Perspektiven für das Systemdesign aufzunehmen.

Die fachkonzeptionellen Überlegungen sowie während des Projekts verwendeten Methoden und Modelle, anknüpfende Konzeptionen und Implementierungen für Smart-Glasses-basierte Anwendungsfälle und deren wirtschaftliche Betrachtung bis hin zu einer Low-Code-Plattform zur Adaption der generierten Erkenntnisse auf weiteren Industrien und Branchen werden in den nachfolgenden Beiträgen dieses Bandes des Forschungsprojekts GLASSHOUSE beschrieben.

\section{Literatur}

Bundesvereinigung Logistik e.V. (2018) Logistikumsatz und Beschäftigung. https://www.bvl.de/service/zahlen-daten-fakten/umsatz-und-beschaeftigung, abgerufen am 14.11.2019.

Caudell TP, Mizell DW (1992) Augmented reality: an application of heads-up display technology to manual manufacturing processes. In: Proceedings of the Twenty-Fifth Hawaii International Conference on System Sciences, 659-669

Czernin J, Schocke KO (2016) Handlungsfelder der Personalarbeit in der Logistik. Studienbericht, Frankfurt University of Applied Science

DHL Trend Research (2014) Augmented Reality in Logistics. https://www.dhl.com/content/dam/downloads/g0/about_us/logistics_insights/csi_augm ented_reality_report_290414.pdf, abgerufen am 04.12.2019

Epson (2019) Aktuelle Übersicht der Smart-Glasses-Modelle der Firma Epson. https://www.epson.de/products/see-through-mobile-viewer/, abgerufen am 04.12.2019

Günthner WA, Blomeyer N, Reif R, Schedlbauer M (2009) Pick-by-Vision: Augmented Reality unterstützte Kommissionierung. In: Günthner WA (Hrsg) fml - Lehrstuhl für Fördertechnik Materialfluss Logistik. Technische Universität München

Helmke B (2019) Digitalisierung in der Logistik. In: Hartel, D (Hrsg) Projektmanagement in Logistik und Supply Chain Management: Praxisleitfaden mit Beispielen aus Industrie, Handel und Dienstleistung. Springer Fachmedien, Wiesbaden

Henderson S, Feiner S (2011) Exploring the benefits of augmented reality documentation for maintenance and repair. Vis. Comput. Graph. IEEE Trans. 17:1355-1368

Kersten W, Seiter M, von See M, Hackitus N, Maurer T (2017) Trends und Strategien in Logistik und Supply Chain Management - Chancen der digitalen Transformation. DVV Media Group GmbH, Hamburg

Knapp AG (2014) Pick-by-Vision-Lösung der Firma Knapp. https://www.knapp.com/en/solutions/technologies/picking/, abgerufen am 04.12.2019

Meier P, Kuhn M, Angermann F (2011) Verfahren zur Darstellung von virtueller Information in einer Ansicht einer realen Umgebung. Patent WO 2011020793 A3

Microsoft (2019) Produktseite der HoloLens. https://www.microsoft.com/de-de/hololens/, abgerufen am 04.12.2019

Niemöller C, Zobel B, Berkemeier L, Metzger D, Werning S, Adelmeyer T, Ickerott I, Thomas, O (2017) Sind Smart Glasses die Zukunft der Digitalisierung von Arbeitsprozessen? Explorative Fallstudien zukünftiger Einsatzszenarien in der Logistik. In: Leimeister JM, Brenner W (Hrsg) 13. International Conference on Wirtschaftsinformatik (WI). St. Gallen, 410-424 
Pcdata (2014) Distrib AR, Augmented Reality - Pick-by-Vision-Lösung der Firma Pcdata. https://www.pcdata-logistics.com/logistics-automation/solutions-products/augmentedreality-distrib-ar/?lang=de, abgerufen am 04.12.2019

Reif R, Walch D (2008) Augmented \& Virtual Reality applications in the field of logistics. The Visual Computer 24:987-994

SAP (2014) Hands-free picking with smart glasses and SAP AR Warehouse Picker. https://www.sap.com/products/augmented-reality-warehouse-app.html, abgerufen am 04.12 .2019

Schroven A (2015) Demographischer Wandel - Herausforderung für die Logistik. In: Voß P (Hrsg) Logistik - eine Industrie, die (sich) bewegt: Strategien und Lösungen entlang der Supply Chain 4.0. Springer Fachmedien, Wiesbaden, 19-29

Stölzle W, Martin J (2016) Logistikinnovation - Auf dem Weg zu Business Innovation in der Logistikdienstleistung. In: Hoffmann CP, Lennerts S, Schmitz C, Stölzle W, Uebernickel F (Hrsg) Business Innovation: Das St. Galler Modell. Springer Fachmedien, Wiesbaden, 499-529

Sutherland IE (1968) A head-mounted three dimensional display. In: Proceedings of the December 9-11, 757-764. ACM, New York, USA

ten Hompel M, Kerner S (2015) Logistik 4.0. Informatik-Spektrum, 38 (3):176-182.

Vuzix (2019) Übersicht aktueller Smart-Glasses-Modelle der Firma Vuzix. https://www.vuzix.com/Products, abgerufen am 04.12.2019

Zobel B, Berkemeier L, Werning S, Thomas O (2016) Augmented Reality am Arbeitsplatz der Zukunft: Ein Usability-Framework für Smart Glasses. In: Mayr HC, Pinzger M (Hrsg) Informatik 2016. Gesellschaft für Informatik e.V., Bonn, 1727-1740 